\title{
Fermi Liquid Theory for Nonlinear Transport through a Multilevel Anderson Impurity
}

\section{Yoshimichi Teratani, Rui Sakano, Akira Oguri}

\begin{tabular}{|c|c|}
\hline Citation & PHYSICAL REVIEW LETTERS. 125(21); 216801 \\
\hline Date & $2020-11-17$ \\
\hline Type & Journal Article \\
\hline Textversion & Publisher \\
\hline Highlights & $\begin{array}{l}\text { ৩ナノスケール素子や磁性原子の電子状態に、新たな量子多体効果が潜むことを数理的 } \\
\text { に解明。 } \\
\text { 〉量子液体状態の理解に新たな視点を与え、新物質や量子情報の分野にも繋がる理論研 } \\
\text { 究。 }\end{array}$ \\
\hline Rights & $\begin{array}{l}\text { C } 2020 \text { American Physical Society. This article may be downloaded for personal use } \\
\text { only. Any other use requires prior permission of American Physical Society. } \\
\text { The following article appeared in PHYSICAL REVIEW LETTERS, Vol. 125, Issu. } \\
21,216801 \text { and may be found at https://doi.org/10.1103/PhysRevLett.125.216801. }\end{array}$ \\
\hline doi & 10.1103/PhysRevLett.125.216801 \\
\hline
\end{tabular}

Self-Archiving by Author(s)

Placed on: Osaka City University

Yoshimichi Teratani, Rui Sakano, and Akira Oguri. (2020). Fermi Liquid Theory for Nonlinear Transport through a Multilevel Anderson Impurity. PHYSICAL REVIEW LETTERS. 125, 216801. doi: 10.1103/PhysRevLett.125.216801 


\begin{tabular}{|c|c|}
\hline Desciption & 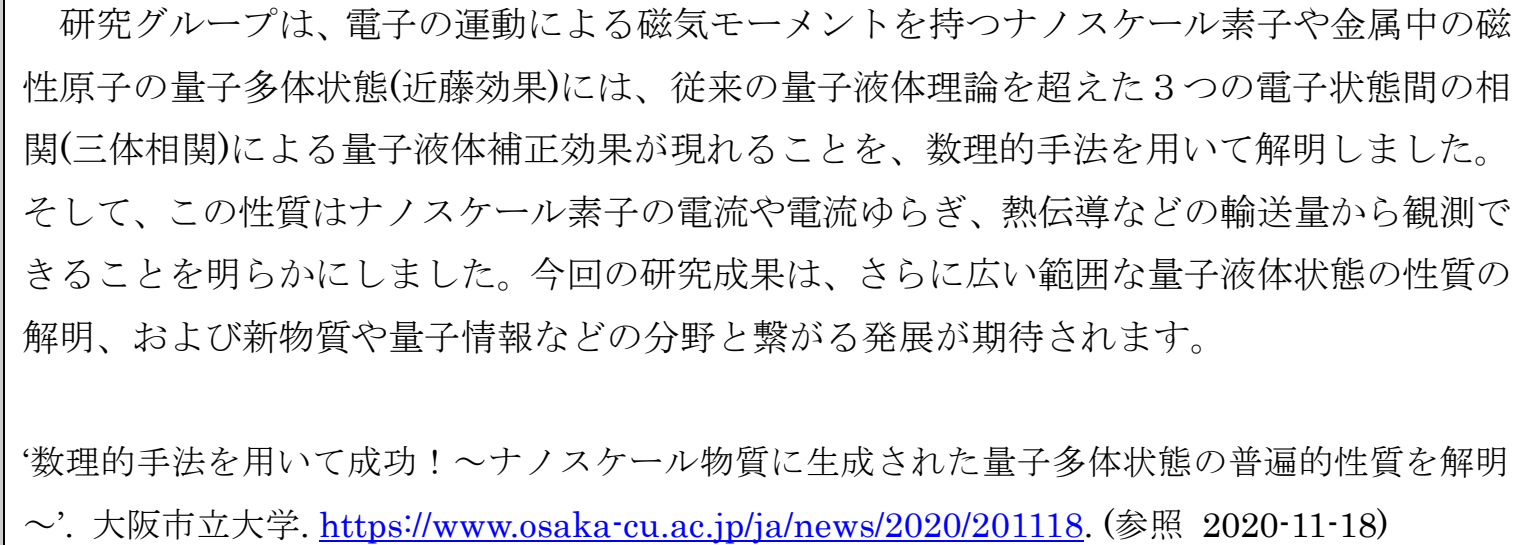 \\
\hline
\end{tabular}




\title{
Fermi Liquid Theory for Nonlinear Transport through a Multilevel Anderson Impurity
}

\author{
Yoshimichi Teratani® \\ Department of Physics, Osaka City University, Sumiyoshi-ku, Osaka 558-8585, Japan \\ Rui Sakano \\ The Institute for Solid State Physics, The University of Tokyo, Kashiwa, Chiba 277-8581, Japan \\ Akira Oguri® \\ Department of Physics, Osaka City University, Sumiyoshi-ku, Osaka 558-8585, Japan \\ and Nambu Yoichiro Institute of Theoretical and Experimental Physics, Osaka City University, Osaka 558-8585, Japan
}

(Received 21 January 2020; accepted 15 October 2020; published 17 November 2020)

\begin{abstract}
We present a microscopic Fermi liquid view on the low-energy transport through an Anderson impurity with $N$ discrete levels, at arbitrary electron filling $N_{d}$. It is applied to nonequilibrium current fluctuations, for which the two-quasiparticle collision integral and the three-body correlations that determine the quasiparticle energy shift play important roles. Using the numerical renormalization group up to $N=6$, we find that for strong interactions the three-body fluctuations are determined by a single parameter other than the Kondo energy scale in a wide filling range $1 \lesssim N_{d} \lesssim N-1$. It significantly affects the current noise for $N>2$ and the behavior of noise in magnetic fields.
\end{abstract}

DOI: 10.1103/PhysRevLett.125.216801

Introduction.-Highly correlated low-energy states of the Kondo systems show fascinating universal behavior [1], which can be described by a Fermi liquid (FL) theory in zero dimension [2-6]. FL behaviors have been observed for the nonlinear current through quantum dots $[7,8]$ and also the current noise [9-12], which is now one of the most important probes to explore quantum states. Furthermore, in addition to the spin, internal degrees of freedom such as orbital, flavor, etc., bring an interesting variety in the Kondo effect, occurring in a carbon nanotube $[12,13]$ and novel quantum systems, such as ultracold atomic gases [14] and quark matters [15].

Transport properties of the local FL have successfully been described by the renormalized quasiparticles and their collisions due to the residual interaction, especially at the symmetric point where both the particle-hole $(\mathrm{PH})$ and time-reversal (TR) symmetries are present [16-19]. These symmetries are broken in real systems by external fields, such as a gate voltage and a magnetic field. In this case, a single quasiparticle captures the quadratic dependence on frequency $\omega$, temperature $T$, and bias voltage $V$, not only through the well-investigated damping rate but also through the energy shift. It has recently been clarified that the quadratic energy shift is determined by the three-body correlations between the impurity electrons [20-24]. It shows that the three-body correlations are essential parameters for describing the FL transport.

Despite its importance, the current noise [25-31] has been still less elucidated away from the symmetric point. A major milestone was achieved by Mora et al. [20], who have extended Nozières phenomenological FL theory [3] to give the formula of the nonlinear noise for a $\mathrm{PH}$ asymmetric single-orbital Anderson model at zero magnetic field. Further investigation, however, is required to clarify the physics of nonequilibrium fluctuations in the Kondo systems with various internal degrees of freedom.

In this Letter, we give a microscopic view on the lowenergy transport through a multilevel Anderson impurity for a wide range of electron fillings $N_{d}$. It is described in terms of five FL parameters, which can be calculated using the numerical renormalization group (NRG) [2] up to $N=6$. We find that for strong interactions the three-body correlations for $N$ degenerate levels are determined by a single parameter over a wide filling range $1 \lesssim N_{d} \lesssim N-1$, which includes the intermediate valence regions. We also provide a current-noise formula for the FL, taking into account all the two-quasiparticle collision processes [32,33]. It satisfies a Ward identity [4-6] for the Keldysh vertex function and resolves an essential problem of the current conservation of the correlated electrons under a nonequilibrium condition $[16,25]$. We also calculate the nonlinear noise using the NRG and demonstrate that the internal degrees of freedom give a wide variety to the filling dependence. We also examine the effect of a magnetic field that breaks the TR symmetry and show that the noise of a spin-1/2 quantum dot exhibits a universal Kondo scaling behavior.

Model.-We consider an $N$-level Anderson impurity coupled to two leads on the left $(L)$ and right $(R)$, 


$$
\begin{aligned}
\mathcal{H}= & \sum_{\sigma=1}^{N} \epsilon_{d \sigma} n_{d \sigma}+\sum_{\lambda=L, R} \sum_{\sigma=1}^{N} v_{\lambda}\left(\psi_{\lambda \sigma}^{\dagger} d_{\sigma}+d_{\sigma}^{\dagger} \psi_{\lambda \sigma}\right) \\
& +\sum_{\lambda=L, R} \sum_{\sigma=1}^{N} \int_{-D}^{D} d \epsilon \epsilon c_{\epsilon \lambda \sigma}^{\dagger} c_{\epsilon \lambda \sigma}+\frac{U}{2} \sum_{\sigma \neq \sigma^{\prime}} n_{d \sigma} n_{d \sigma^{\prime}},
\end{aligned}
$$

where $d_{\sigma}^{\dagger}$ creates an impurity electron with energy $\epsilon_{d \sigma}, n_{d \sigma} \equiv d_{\sigma}^{\dagger} d_{\sigma}$, and $U$ is the Coulomb repulsion. Conduction electrons are normalized as $\left\{c_{\epsilon \lambda \sigma}, c_{\epsilon^{\prime} \lambda^{\prime} \sigma^{\prime}}^{\dagger}\right\}=$ $\delta_{\lambda \lambda^{\prime}} \delta_{\sigma \sigma^{\prime}} \delta\left(\epsilon-\epsilon^{\prime}\right)$. The coupling $v_{\lambda}$ between $\psi_{\lambda \sigma} \equiv$ $\int_{-D}^{D} d \epsilon \sqrt{\rho_{c}} c_{\epsilon \lambda \sigma}$ and $d_{\sigma}^{\dagger}$ yields a resonance of the width $\Delta \equiv \Gamma_{L}+\Gamma_{R}$, with $\Gamma_{\lambda}=\pi \rho_{c} v_{\lambda}^{2}, \rho_{c}=1 /(2 D)$, and $D$ as the half band width.

In this Letter, we study the nonlinear current noise [25]

$$
S_{\mathrm{noise}}^{\mathrm{QD}}=\int_{-\infty}^{\infty} d t\langle\delta \hat{J}(t) \delta \hat{J}(0)+\delta \hat{J}(0) \delta \hat{J}(t)\rangle_{V}
$$

Here, $\delta \hat{J}(t) \equiv \hat{J}(t)-\langle\hat{J}(0)\rangle_{V}$ is the current fluctuation operator through the quantum dot (QD) [34], and $\langle\cdots\rangle_{V}$ is the Keldysh steady-state average defined at finite bias voltages $e V \equiv \mu_{L}-\mu_{R}$ with $\mu_{\lambda}$ the chemical potential for $\lambda=L, R$ (see Supplemental Material [35]). The average current $J \equiv\langle\hat{J}(0)\rangle_{V}$ is given by [16]

$$
J=\frac{e}{h} \sum_{\sigma} \int_{-\infty}^{\infty} d \omega\left[f_{L}(\omega)-f_{R}(\omega)\right] \mathcal{T}_{\sigma}(\omega) .
$$

Here, $f_{\lambda}(\omega) \equiv\left[e^{\left(\omega-\mu_{\lambda}\right) / T}+1\right]^{-1}$ is the Fermi function, $\mathcal{T}_{\sigma}(\omega) \equiv-\left[4 \Gamma_{L} \Gamma_{R} /\left(\Gamma_{L}+\Gamma_{R}\right)\right] \operatorname{Im} G_{\sigma}^{r}(\omega)$ is the transmission probability, $G_{\sigma}^{r}(\omega)=\left[\omega-\epsilon_{d \sigma}+i \Delta-\Sigma_{\sigma}^{r}(\omega)\right]^{-1}$ is the retarded Green's function and $\Sigma_{\sigma}^{r}(\omega)$ is the self-energy. From this $\mathcal{T}_{\sigma}(\omega)$, we can also deduce the thermal conductivity $\kappa_{\mathrm{QD}}[36]$ for the heat current $J_{Q}=-\kappa_{\mathrm{QD}} \delta T$, induced by the temperature difference $\delta T$ between the two leads [37].

Fermi liquid parameters. - We investigate low-energy transport up to next leading order. To this end, we expand $\Sigma_{\sigma}^{r}(\omega)$ up to terms of order $\omega^{2}, T^{2}$, and $(e V)^{2}$ for general $N$, extending the latest FL description for spin-1/2 case $[22,23]$. The expansion coefficients play an important role as the FL parameters.

The phase shift $\delta_{\sigma} \equiv \cot ^{-1}\left(\epsilon_{d \sigma}^{*} / \Delta\right)$ is a parameter of primary importance, with $\epsilon_{d \sigma}^{*} \equiv \epsilon_{d \sigma}+\left.\Sigma_{\sigma}^{r}(0)\right|_{T=e V=0}$ the effective impurity level. It determines the occupation number $\left\langle n_{d \sigma}\right\rangle=\delta_{\sigma} / \pi$ and the density of states $\rho_{d \sigma} \equiv \sin ^{2} \delta_{\sigma} /(\pi \Delta)$. The renormalization factor is given by the first derivative $z_{\sigma} \equiv\left\{1-\left.\left[\partial \Sigma_{\sigma}^{r}(\omega) / \partial \omega\right]\right|_{\omega=0}\right\}^{-1}$, defined at $T=e V=0$. It is also related to the static susceptibility $\chi_{\sigma_{1} \sigma_{2}} \equiv \int_{0}^{1 / T} d \tau\left\langle\delta n_{d \sigma_{1}}(\tau) \delta n_{d \sigma_{2}}\right\rangle$, as $\chi_{\sigma \sigma} \stackrel{T \rightarrow 0}{\rightarrow} \rho_{d \sigma} / z_{\sigma}$, with $\delta n_{d \sigma} \equiv n_{d \sigma}-\left\langle n_{d \sigma}\right\rangle$ [4-6]. The second derivative is a complex number, the imaginary part of which corresponds to the single-quasiparticle damping rate of order $\omega^{2}, T^{2}$, and $(e V)^{2}[16,17]$. The real part corresponds to the quadratic energy shift that is determined by the nonlinear susceptibility defined at equilibrium [22,23],

$\chi_{\sigma_{1} \sigma_{2} \sigma_{3}}^{[3]} \equiv-\int_{0}^{1 / T} d \tau_{3} \int_{0}^{1 / T} d \tau_{2}\left\langle T_{\tau} \delta n_{d \sigma_{3}}\left(\tau_{3}\right) \delta n_{d \sigma_{2}}\left(\tau_{2}\right) \delta n_{d \sigma_{1}}\right\rangle$,

with $T_{\tau}$ the imaginary-time ordering operator. It can also be written as $\chi_{\sigma_{1} \sigma_{2} \sigma_{3}}^{[3]}=\partial \chi_{\sigma_{1} \sigma_{2}} / \partial \epsilon_{d \sigma_{3}}$ and contributes to the transport when the PH or TR symmetry is broken.

$\mathrm{SU}(N)$ symmetric case.-In the case at which the $N$ impurity levels are degenerate $\epsilon_{d \sigma} \equiv \epsilon_{d}$, the linear susceptibility $\chi_{\sigma \sigma^{\prime}}$ has only two independent components. The diagonal element determines the energy scale $T^{*} \equiv 1 /\left(4 \chi_{\sigma \sigma}\right)$, by which the $T$-linear specific heat is scaled as $\mathcal{C}_{\text {imp }}=\left(N \pi^{2} / 12\right)\left(T / T^{*}\right)$. It can also be identified as the Kondo temperature in the strong-coupling limit. The other one is the off-diagonal element $\chi_{\sigma \sigma^{\prime}}$ for $\sigma \neq \sigma^{\prime}$, which is related to the Wilson ratio $R \equiv 1-\chi_{\sigma \sigma^{\prime}} / \chi_{\sigma \sigma}$ [38]. Similarly, the nonlinear susceptibility has three independent components for $N \geq 3$ : the diagonal element $\chi_{\sigma \sigma \sigma}^{[3]}$ and two off-diagonal ones, which can also be expressed in the following form for $\sigma \neq \sigma^{\prime} \neq \sigma^{\prime \prime} \neq \sigma$ :

$$
\begin{gathered}
-(N-1) \chi_{\sigma \sigma^{\prime} \sigma^{\prime}}^{[3]}=\chi_{\sigma \sigma \sigma}^{[3]}-\frac{\partial \chi_{\sigma \sigma}}{\partial \epsilon_{d}}, \\
\frac{(N-1)(N-2)}{2} \chi_{\sigma \sigma^{\prime} \sigma^{\prime \prime}}^{[3]}=\chi_{\sigma \sigma \sigma}^{[3]}-\frac{\partial \chi_{\sigma \sigma}}{\partial \epsilon_{d}}+\frac{N-1}{2} \frac{\partial \chi_{\sigma \sigma^{\prime}}}{\partial \epsilon_{d}} .
\end{gathered}
$$

In this Letter, we obtain the low-energy expansion of $S_{\text {noise }}^{\mathrm{QD}}, J$, and $\kappa_{\mathrm{QD}}$ up to next leading order, specifically for symmetric junctions $\Gamma_{L}=\Gamma_{R}$ and $\mu_{L}=-\mu_{R}=e V / 2$,

$$
\begin{aligned}
S_{\text {noise }}^{\mathrm{QD}} & =\frac{2 N e^{2}|e V|}{h}\left[\frac{\sin ^{2} 2 \delta}{4}+C_{S}\left(\frac{e V}{T^{*}}\right)^{2}+\cdots\right], \\
\frac{d J}{d V} & =\frac{N e^{2}}{h}\left[\sin ^{2} \delta-C_{T}\left(\frac{\pi T}{T^{*}}\right)^{2}-C_{V}\left(\frac{e V}{T^{*}}\right)^{2}+\cdots\right], \\
\kappa_{\mathrm{QD}} & =\frac{N \pi^{2} T}{3 h}\left[\sin ^{2} \delta-C_{\kappa}^{\mathrm{QD}}\left(\frac{\pi T}{T^{*}}\right)^{2}+\cdots\right] .
\end{aligned}
$$

The explicit expressions of the coefficients $C_{S}, C_{V}, C_{T}$, and $C_{\kappa}^{\mathrm{QD}}$ are listed in Table I. Each of these $C$ 's consists of two parts, denoted as $W$ and $\Theta$. The $W$ part represents two-body contributions, which can be described in terms of $R$ and $\delta$. The $\Theta$ part represents dimensionless three-body contributions,

$$
\Theta_{\mathrm{I}} \equiv \frac{\sin 2 \delta}{2 \pi} \frac{\chi_{\sigma \sigma \sigma}^{[3]}}{\chi_{\sigma \sigma}^{2}}, \quad \Theta_{\mathrm{II}} \equiv \frac{\sin 2 \delta}{2 \pi} \frac{\chi_{\sigma \sigma^{\prime} \sigma^{\prime}}^{[3]}}{\chi_{\sigma \sigma}^{2}} .
$$

Therefore, the low-energy transport of the $\mathrm{SU}(N)$ Fermi liquid are determined completely by five parameters: $\delta, T^{*}$, $R, \Theta_{\mathrm{I}}$, and $\Theta_{\mathrm{II}}$. These FL parameters can also be deduced experimentally through measurements of the coefficients $C$ 's. We note that another parameter for three different 
TABLE I. Coefficients $C$ 's introduced in Eq. (5). W's and $\Theta$ 's represent the two- and three-body contributions, respectively.

\begin{tabular}{ll}
\hline \hline$C_{S}=\left(\pi^{2} / 192\right)\left[W_{S}-\cos 2 \delta\left\{\Theta_{\mathrm{I}}+3(N-1) \Theta_{\mathrm{II}}\right\}\right]$ & $W_{S} \equiv \cos 4 \delta+\left[4+5 \cos 4 \delta+\frac{3}{2}(1-\cos 4 \delta)(N-2)\right](N-1)(R-1)^{2}$ \\
$C_{V}=\left(\pi^{2} / 64\right)\left[W_{V}+\Theta_{\mathrm{I}}+3(N-1) \Theta_{\mathrm{II}}\right]$ & $W_{V} \equiv-\left[1+5(N-1)(R-1)^{2}\right] \cos 2 \delta$ \\
$C_{T}=\left(\pi^{2} / 48\right)\left[W_{T}+\Theta_{\mathrm{I}}+(N-1) \Theta_{\mathrm{II}}\right]$ & $W_{T} \equiv-\left[1+2(N-1)(R-1)^{2}\right] \cos 2 \delta$ \\
$C_{\kappa}^{\mathrm{QD}}=\left(7 \pi^{2} / 80\right)\left[W_{\kappa}^{\mathrm{QD}}+\Theta_{\mathrm{I}}+\frac{5}{21}(N-1) \Theta_{\mathrm{II}}\right]$ & $W_{\kappa}^{\mathrm{QD}} \equiv[(10-11 \cos 2 \delta) / 21]-\frac{6}{7}(N-1)(R-1)^{2} \cos 2 \delta$ \\
\hline \hline
\end{tabular}

levels, $\Theta_{\mathrm{III}} \equiv(\sin 2 \delta / 2 \pi)\left(\chi_{\sigma \sigma^{\prime} \sigma^{\prime \prime}}^{[3]} / \chi_{\sigma \sigma}^{2}\right)$, does not affect $C$ 's for symmetric junctions. Nevertheless, it contributes to the transport for $N \geq 3$ when the tunneling couplings or the chemical potentials are asymmetric.

The nonlinear noise of the Fermi liquid is determined not only by a single-quasiparticle excitation but also by twoquasiparticle collisions described by the Keldysh vertex corrections [25]. In this Letter, we calculate the vertex function up to order $e V$ [35], extending the diagrammatic approach of Yamada-Yosida [4-6]. Consequently, the collision contributions $C_{S}^{\text {coll }}$ and the single-quasiparticle ones $C_{S}^{\mathrm{qp}}$ yield the nonlinear noise $C_{S}=C_{S}^{\mathrm{qp}}+C_{S}^{\text {coll }}$,

$$
C_{S}^{\text {coll }}=\left[\frac{7+5 \cos 4 \delta}{2}+\frac{3}{2}(1-\cos 4 \delta)(N-2)\right] \frac{\tilde{K}^{2}}{N-1},
$$

with $\tilde{K} \equiv(N-1)(R-1)$. The second term in the bracket emerges through the collisions specific to multilevel impurities for $N>2$, and it vanishes in the $\mathrm{SU}(2)$ symmetric case or the PH symmetric case at which $\delta=\pi / 2$.

Filling dependence of the FL state.-How does the FL state evolve as the number of levels $N$ and their position $\epsilon_{d}$ vary? As the electron configuration $N_{d} \equiv \sum_{\sigma}\left\langle n_{d \sigma}\right\rangle$ continuously varies with $\epsilon_{d}$, a different class of the Kondo and valence fluctuation states emerge for multilevel systems $N>2$. To our knowledge, however, the behavior of threebody correlations $\Theta$ 's that determine the nonlinear transport has not been explored so much, whereas the two-body correlations have been well investigated for $N=4$ $[30,39,40]$. In this Letter, we calculate the FL parameters for $N=4,6$ with the NRG, using the interleaved algorithm particularly for $N=6$ [41]. To be specific, we choose the Coulomb interaction to be much larger than the hybridization energy scale: $U /(\pi \Delta)=5.0$. The results are plotted vs $\xi_{d} \equiv \epsilon_{d}+(N-1) U / 2$ in Fig. 1 for (left panels) $N=4$ and (right panels) $N=6$.

The top panels of Fig. 1 show the two-body correlations, relating to $\left\langle n_{d \sigma}\right\rangle, \chi_{\sigma \sigma}$, and $\chi_{\sigma \sigma^{\prime}}$. We see that $\sin ^{2} \delta$, which determines $\mathcal{T}_{\sigma}(0)$ at $T=e V=0$, shows a flat Kondo ridge of the unitary limit $\delta \simeq \pi / 2$ near the PH symmetric point $\left|\xi_{d}\right| \lesssim U / 2$ where the occupation number is almost locked at $N_{d} \simeq N / 2$. The other Kondo ridges also emerge at $\xi_{d}$ where $N_{d}$ approaches an integer: $\xi_{d} \simeq \pm U$ for $N=4$, and also $\xi_{d} \simeq \pm U, \pm 2 U$ for $N=6$.

The renormalization factor $z$, which determines the energy scale $T^{*}=z \pi \Delta /\left(4 \sin ^{2} \delta\right)$, is also shown in the top panels. It is significantly suppressed over a wide range of gate voltages $\left|\xi_{d}\right| \lesssim[(N-1) / 2] U$, and appears as a broad valley. This valley becomes shallow as $N$ increases and vanishes in the large $N$ limit [40]. Inside the valley, $z$ has minimums at $\xi_{d} \simeq[(N-2 M) / 2] U$ for $M=1,2, \ldots, N-1$, where the occupation number approaches an integer $N_{d}=M$. At these minimums, the low-energy states can be described by the $\mathrm{SU}(N)$ Kondo model in the strong-coupling limit $U \gg \Delta$. We find that $z$ is also suppressed at local maximums corresponding to the intermediate valence states, for both $N=4$ and 6. In the top panels, the rescaled Wilson ratio $\tilde{K}$ is also shown. It is almost saturated to the universal value $\tilde{K}=1$ and its derivative becomes very small $\partial \tilde{K} / \partial \epsilon_{d} \sim 0$ in the whole region of the broad valley $1 \lesssim N_{d} \lesssim N-1$. It reveals the fact that not only the charge susceptibility $\chi_{c} \equiv$ $-\partial\left\langle n_{d \sigma}\right\rangle / \partial \epsilon_{d}=\chi_{\sigma \sigma}(1-\tilde{K})$ but its derivative $\partial \chi_{c} / \partial \epsilon_{d}$ is suppressed in this region.
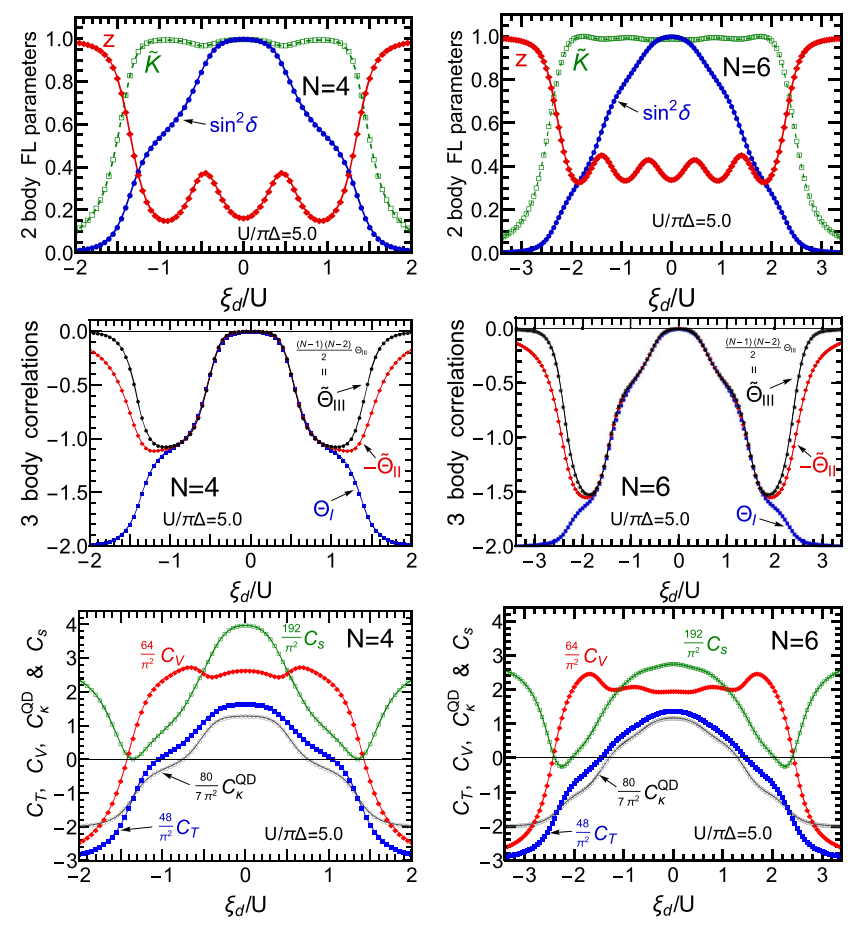

FIG. 1. Fermi liquid parameters for $\mathrm{SU}(N)$ Anderson model are plotted vs $\xi_{d} \equiv \epsilon_{d}+(N-1) U / 2$ for $U /(\pi \Delta)=5.0, N=4$ (left column) and $N=6$ (right column). Top row: $\sin ^{2} \delta$, renormalization factor $z$, and $\tilde{K} \equiv(N-1)(R-1)$. Middle row: $\Theta_{\mathrm{I}}$, $-\tilde{\Theta}_{\mathrm{II}} \equiv-(N-1) \Theta_{\mathrm{II}}, \quad$ and $\quad \tilde{\Theta}_{\mathrm{III}} \equiv\{[(N-1)(N-2)] / 2\} \Theta_{\mathrm{III}}$. Bottom row: $\left(48 / \pi^{2}\right) C_{T}, \quad\left(64 / \pi^{2}\right) C_{V}, \quad\left(80 / 7 \pi^{2}\right) C_{\kappa}^{\mathrm{QD}}$, and $\left(192 / \pi^{2}\right) C_{S}$. 
The three-body correlation $\Theta_{\mathrm{I}}$ is plotted in the middle panels of Fig. 1, together with the other two rescaled ones: $-\tilde{\Theta}_{\text {II }} \equiv-(N-1) \Theta_{\text {II }}$ and $\tilde{\Theta}_{\text {III }} \equiv\{[(N-1)(N-2)] / 2\} \Theta_{\text {III }}$. These $\Theta$ 's also show plateau structures due to the Kondo effect at the values of $\xi_{d}$ corresponding to integer $N_{d}$ and almost vanish at $\left|\xi_{d}\right| \lesssim U / 2$. We find that these three parameters $\Theta_{\mathrm{I}},-\tilde{\Theta}_{\mathrm{II}}$, and $\tilde{\Theta}_{\mathrm{III}}$ approach each other very closely over a wide gate-voltage range $\left|\xi_{d}\right| \lesssim[(N-2) / 2] U$, at which $1 \lesssim N_{d} \lesssim N-1$. This indicates that contributions of the diagonal element $\chi_{\sigma \sigma \sigma}^{[3]}$ dominate the terms in the righthand side of Eq. (4); i.e., $\chi_{\sigma \sigma \sigma}^{[3]}$ becomes much greater than $\partial \chi_{\sigma \sigma} / \partial \epsilon_{d}$ and $[(N-1) / 2]\left(\partial \chi_{\sigma \sigma^{\prime}} / \partial \epsilon_{d}\right)$. It also reveals the fact that not only $\partial \chi_{c} / \partial \epsilon_{d}$ but also $\partial \chi_{s} / \partial \epsilon_{d}$, the derivative of the spin susceptibility $\chi_{s} \propto \chi_{\sigma \sigma}-\chi_{\sigma \sigma^{\prime}}$, becomes much smaller than $\left(T^{*}\right)^{-2}$. Thus, for large $U$, the FL properties are characterized by three parameters $\delta, T^{*}$, and $\Theta_{\mathrm{I}}$ over the wide filling range $1 \lesssim N_{d} \lesssim N-1$. Outside this region, the $\Theta$ 's approach the noninteracting values: $\Theta_{\mathrm{I}} \rightarrow-2$, and the other two vanish as $\left|\xi_{d}\right| \rightarrow \infty$.

Nonequilibrium FL fluctuations.-We show in the following how the transport coefficients evolve as $N_{d}$ varies continuously. The NRG results are also plotted in Fig. 1. The difference between the $C$ 's near half filling $\left|\xi_{d}\right| \lesssim U / 2$ is caused by the two-body contributions $W$ 's as the $\Theta$ 's almost vanish. In particular, the $T^{2}$ conductance $C_{T}$ is determined by $W_{T}$ over the wide filling range $1 \lesssim N_{d} \lesssim$ $N-1$ as the three-body contributions almost cancel out $\Theta_{\mathrm{I}}+(N-1) \Theta_{\mathrm{II}} \approx 0$, reflecting the suppression of $\partial \chi_{c} / \partial \epsilon_{d}$ and $\partial \chi_{s} / \partial \epsilon_{d}$ mentioned above. For the thermal conductivity, the three-body contributions become negative in this region, $\Theta_{\mathrm{I}}+\frac{5}{21}(N-1) \Theta_{\mathrm{II}} \approx-\frac{16}{21} \tilde{\Theta}_{\mathrm{II}}$, but otherwise $C_{\kappa}^{\mathrm{QD}}$ shows a similar $\xi_{d}$ dependence to that of $C_{T}$. The threebody contributions on the $(\mathrm{eV})^{2}$ conductance $C_{V}$ are given by $\Theta_{V} \equiv \Theta_{\text {I }}+3(N-1) \Theta_{\text {II }}$, which takes a value $\Theta_{V} \approx 2 \tilde{\Theta}_{\text {II }}$ in the same filling range. Thus, $C_{V}$ is significantly enhanced at $N_{d} \simeq 1$ and $N-1$ where $-\tilde{\Theta}_{\text {II }}$ shows a deep valley. It pushes the tail of the $C_{V}$ curve outside than that of the $C_{T}$ in the valence fluctuation region toward the empty or fully occupied limit.

The $|e V|^{3}$ current noise also exhibits the Kondo plateau structures as shown in Fig. 1. For $C_{S}$, the three-body contributions enter through $\Theta_{V}$ with a sinusoidal factor: $-\Theta_{V} \cos 2 \delta \approx(\sin 4 \delta / 2 \pi)\left(\chi_{\sigma \sigma \sigma}^{[3]} / \chi_{\sigma \sigma}^{2}\right)$ over the range of $1 \lesssim N_{d} \lesssim N-1$. In the valence fluctuation regions mentioned above, $C_{S}$ has a minimum caused by the higherharmonic $\sin 4 \delta$ and $\cos 4 \delta$ contributions. We also find that $C_{S}$ approaches zero almost simultaneously with $C_{V}$ at $\left|\xi_{d}\right| \simeq 1.4 U$ for $N=4$, and at $\left|\xi_{d}\right| \simeq 2.4 U$ for $N=6$. This proximity of the zero points affects the behavior of an extended Fano factor $F_{K}$, defined as the ratio of order $(\mathrm{eV})^{3}$ current noise to the nonlinear current $[20,29,35]$,

$$
F_{K} \equiv \lim _{|e V| \rightarrow 0} \frac{S_{\text {noise }}^{\mathrm{QD}}-\frac{2 N e^{2}|e V|}{h} \frac{\sin ^{2} 2 \delta}{4}}{-2|e|\left(J-\frac{N e^{2}|V|}{h} \sin ^{2} \delta\right)}=\frac{C_{S}}{C_{V} / 3} .
$$
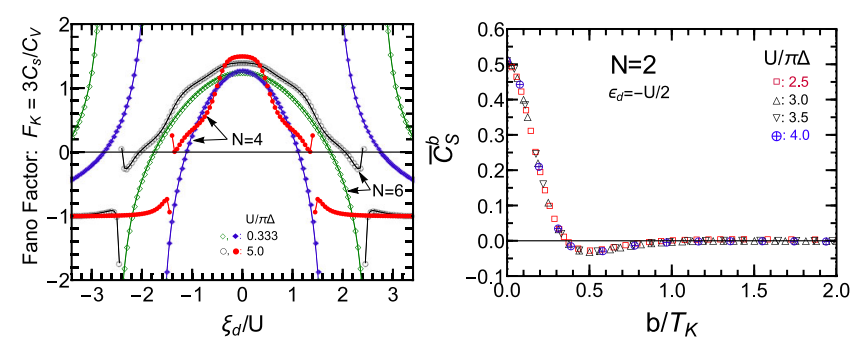

FIG. 2. Nonlinear current-current correlations. Left: $F_{K} \equiv$ $\left[C_{S} /\left(C_{V} / 3\right)\right]$ vs $\xi_{d} / U$ for $\mathrm{SU}(N)$ symmetric case for $N=4$ $(\bullet, \diamond)$ and $N=6(\circ, \diamond)$, for $U /(\pi \Delta)=1 / 3$ (diamonds) and $U /(\pi \Delta)=5$ (circles). Right: $\bar{C}_{S}^{b}$ vs $b / T_{K}$ for $N=2$ at half filling $\epsilon_{d}=-U / 2$, for $U /(\pi \Delta)=2.5,3.0,3.5$, 4.0, with $b$ the magnetic field and $T_{K}$ the Kondo temperature at $b=0$.

This formula for the $\mathrm{SU}(N)$ Anderson model includes the result of Mora et al., obtained for $N=2$ at zero magnetic field [20], as a special case. In the strong-coupling limit at integer $N_{d}$, it also agrees with another noise formula of Mora et al. for the $\mathrm{SU}(N)$ Kondo model [29].

The Fano factor for $N=4,6$ is plotted vs $\xi_{d}$ for two different values of $U$ in the left panel of Fig. 2. It reaches the local maximum $F_{K} \rightarrow\left(N-1+9 \tilde{K}^{2}\right) /\left(N-1+5 \tilde{K}^{2}\right)$ at $\xi_{d}=0$ [30] and has positive plateaus for large $U$ at integer $N_{d}$. In the limit of $\left|\xi_{d}\right| \rightarrow \infty$, the ratio becomes negative and takes the noninteracting value $F_{K} \rightarrow-1$. By definition, $F_{K}$ changes sign at the zero points of $C_{S}$. It also diverges at the zero point of $C_{V}$, where the nonlinear component of $J$ changes direction from backward to forward. Such a singularity already exists for $U=0$ at $\left|\xi_{d}\right|=\Delta / \sqrt{3}$. For large $U$, $F_{K}$ diverges near $\left|\xi_{d}\right| \simeq[(N-1) / 2] U$ in the valence fluctuation region toward the empty or fully occupied limit. We can see that sign of the coefficient $C_{S}$ at the singular points becomes positive for large $U$, whereas it is negative for small $U$. Sign change occurs, for both $N=4$ and 6 , at a finite $U$ between the two examined cases $U /(\pi \Delta)=1 / 3$ and 5. It is associated with the large enhancement of three-body contributions $\Theta_{V}$ occurring in the Kondo regime at $N_{d} \simeq 1$ and $N-1$ for $N>2$. In contrast, the NRG calculations examined so far indicate that sign is always negative in the $\mathrm{SU}(2)$ case for any $U \geq 0[20,35]$. The main difference is that in the $\mathrm{SU}(2)$ case the three-body correlations evolve in the valence fluctuation region where electron correlations become less important and the two-body contributions $W_{S}$ dominate $C_{S}$ near the singular point.

Magnetic-field dependence.-We next consider effects of a magnetic field $b$ that breaks the $\mathrm{SU}(N)$ and TR symmetries, specifically, at half filling for $N=2$, where $\epsilon_{d \uparrow}=-U / 2-b, \epsilon_{d \downarrow}=-U / 2+b$, and the electron filling is fixed at $\left\langle n_{d \uparrow}\right\rangle+\left\langle n_{d \downarrow}\right\rangle=1$. In this case, the transport coefficients can be described also by five FL parameters: magnetization $m_{d} \equiv\left\langle n_{d \uparrow}\right\rangle-\left\langle n_{d \downarrow}\right\rangle$, susceptibilities $\chi_{\uparrow \uparrow}=$ $\chi_{\downarrow \downarrow}$ and $\chi_{\uparrow \downarrow}=\chi_{\downarrow \uparrow}$, and three-body correlations $\chi_{\uparrow \uparrow \uparrow}^{[3]}=$ $-\chi_{\downarrow \downarrow \downarrow}^{[3]}$ and $\chi_{\uparrow \downarrow \downarrow}^{[3]}=-\chi_{\uparrow \uparrow \downarrow}^{[3]}$. The nonlinear current for this 
case has previously been studied [21-23]. However, behavior of its fluctuations has not been clarified so far.

Here, we examine the current noise at $T=0$ [42],

$$
S_{\text {noise }}^{\mathrm{QD}}=\frac{4 e^{2}|e V|}{h}\left[\frac{\sin ^{2}\left(\pi m_{d}\right)}{4}+\bar{C}_{S}^{b}\left(\frac{e V}{T_{K}}\right)^{2}+\cdots\right] .
$$

Note that the second term is scaled by $\left.T_{K} \equiv T^{*}\right|_{b=0}$, the Kondo temperature defined at zero field. Thus, the coefficient $\bar{C}_{S}^{b}$ includes all effects of $b$, which enter through the FL parameters. In the right panel of Fig. 2, NRG results for $\bar{C}_{S}^{b}$ are plotted as a function of $b / T_{K}$ for several different values of $U$. We find that the nonlinear noise exhibits a universal behavior for $U /(\pi \Delta) \gtrsim 2.0$ in a similar way that the nonlinear current shows [22,23]. It decreases rapidly as $b$ increases for small fields, changes sign at $b \approx 0.36 T_{K}$, takes a minimum at $b \approx 0.5 T_{K}$, and then approaches zero at $b \gtrsim T_{K}$. We note that order $T^{3}$ thermal conductivity also exhibits the scaling behavior (see Supplemental Material [35]). These observations reflect the fact that the three-body fluctuations show the universal scaling behavior in the Kondo regime without the TR symmetry.

Conclusion.-Nonlinear transport through the $\mathrm{SU}(N)$ Anderson impurity has been described in a unified way with five FL parameters. We have demonstrated how the FL state evolves as electron filling $N_{d}$ varies, using the NRG up to $N=6$. For strong interactions $U$, not only charge fluctuations but also the derivatives of charge and spin susceptibilities are suppressed over a wide filling range $1 \lesssim N_{d} \lesssim N-1$. It reduces the number of variable FL parameters from five to three and causes the Kondo plateau structures emerging for all the coefficients $C$ 's. In particular, the three-body contributions on $C_{V}$ are significantly enhanced at $N_{d} \simeq 1$ and $N-1$ for $N>2$. It also affects the behavior of nonlinear Fano factor $F_{K}$ in the valence fluctuation region. We have also shown that the nonlinear current noise exhibits the universal magnetic-field scaling in the Kondo regime. The FL parameters can also be deduced from experiments and can be used to predict behaviors of unmeasured observables.

We would like to thank K. Kobayashi, T. Hata, M. Ferrier, R. Deblock, and A.C. Hewson for valuable discussions. This work was supported by JSPS KAKENHI Grants No. JP18J10205, No. JP18K03495, and No. JP26220711 and JST CREST Grant No. JPMJCR1876.

[1] A. C. Hewson, The Kondo Problem to Heavy Fermions (Cambridge University Press, Cambridge, England, 1993).

[2] K. G. Wilson, Rev. Mod. Phys. 47, 773 (1975).

[3] P. Nozières, J. Low Temp. Phys. 17, 31 (1974).

[4] K. Yamada, Prog. Theor. Phys. 54, 316 (1975).

[5] H. Shiba, Prog. Theor. Phys. 54, 967 (1975).

[6] A. Yoshimori, Prog. Theor. Phys. 55, 67 (1976).
[7] M. Grobis, I. G. Rau, R. M. Potok, H. Shtrikman, and D. Goldhaber-Gordon, Phys. Rev. Lett. 100, 246601 (2008).

[8] G. D. Scott, Z. K. Keane, J. W. Ciszek, J. M. Tour, and D. Natelson, Phys. Rev. B 79, 165413 (2009).

[9] O. Zarchin, M. Zaffalon, M. Heiblum, D. Mahalu, and V. Umansky, Phys. Rev. B 77, 241303(R) (2008).

[10] T. Delattre, C. Feuillet-Palma, L. G. Herrmann, P. Morfin, J.-M. Berroir, G. Fève, B. Plaçais, D. C. Glattli, M.-S. Choi, C. Mora, and T. Kontos, Nat. Phys. 5, 208 (2009).

[11] Y. Yamauchi, K. Sekiguchi, K. Chida, T. Arakawa, S. Nakamura, K. Kobayashi, T. Ono, T. Fujii, and R. Sakano, Phys. Rev. Lett. 106, 176601 (2011).

[12] M. Ferrier, T. Arakawa, T. Hata, R. Fujiwara, R. Delagrange, R. Weil, R. Deblock, R. Sakano, A. Oguri, and K. Kobayashi, Nat. Phys. 12, 230 (2016).

[13] E. A. Laird, F. Kuemmeth, G. A. Steele, K. Grove-Rasmussen, J. Nygård, K. Flensberg, and L. P. Kouwenhoven, Rev. Mod. Phys. 87, 703 (2015).

[14] K. Ono, J. Kobayashi, Y. Amano, K. Sato, and Y. Takahashi, Phys. Rev. A 99, 032707 (2019).

[15] S. Yasui and S. Ozaki, Phys. Rev. D 96, 114027 (2017).

[16] S. Hershfield, J. H. Davies, and J. W. Wilkins, Phys. Rev. B 46, 7046 (1992).

[17] A. Oguri, Phys. Rev. B 64, 153305 (2001).

[18] A. A. Aligia, J. Phys. Condens. Matter 24, 015306 (2012).

[19] E. Muñoz, C. J. Bolech, and S. Kirchner, Phys. Rev. Lett. 110, 016601 (2013).

[20] C. Mora, C. P. Moca, J. von Delft, and G. Zaránd, Phys. Rev. B 92, 075120 (2015).

[21] M. Filippone, C. P. Moca, A. Weichselbaum, J. von Delft, and C. Mora, Phys. Rev. B 98, 075404 (2018).

[22] A. Oguri and A. C. Hewson, Phys. Rev. Lett. 120, 126802 (2018).

[23] A. Oguri and A.C. Hewson, Phys. Rev. B 97, 035435 (2018).

[24] A. Oguri and A. C. Hewson, Phys. Rev. B 98, 079905(E) (2018).

[25] S. Hershfield, Phys. Rev. B 46, 7061 (1992).

[26] A. O. Gogolin and A. Komnik, Phys. Rev. Lett. 97, 016602 (2006).

[27] E. Sela, Y. Oreg, F. von Oppen, and J. Koch, Phys. Rev. Lett. 97, 086601 (2006).

[28] E. Sela and J. Malecki, Phys. Rev. B 80, 233103 (2009).

[29] C. Mora, P. Vitushinsky, X. Leyronas, A. A. Clerk, and K. Le Hur, Phys. Rev. B 80, 155322 (2009).

[30] R. Sakano, T. Fujii, and A. Oguri, Phys. Rev. B 83, 075440 (2011).

[31] D. B. Karki, C. Mora, J. von Delft, and M. N. Kiselev, Phys. Rev. B 97, 195403 (2018).

[32] L. P. Pitaevskii and E. M. Lifshitz, Physical Kinetics (Butterworth-Heinemann, London, 1981).

[33] H. Haug and A.-P. Jauho, Quantum Kinetics in Transport and Optics of Semiconductors (Springer, New York, 2008).

[34] $\hat{J} \equiv\left(\Gamma_{L} \hat{J}_{R}+\Gamma_{R} \hat{J}_{L}\right) /\left(\Gamma_{L}+\Gamma_{R}\right)$, with $\hat{J}_{\lambda}$ the current flowing between the dot and lead on $\lambda(=L, R)$ side.

[35] See Supplemental Material at http://link.aps.org/supplemental/ 10.1103/PhysRevLett.125.216801 for a more precise formulation and NRG calculations. 
[36] $\kappa_{\mathrm{QD}} \equiv(1 / h T)\left[\sum_{\sigma} \mathcal{L}_{2, \sigma}^{\mathrm{QD}}-\left(\sum_{\sigma} \mathcal{L}_{1, \sigma}^{\mathrm{QD}}\right)^{2} /\left(\sum_{\sigma} \mathcal{L}_{0, \sigma}^{\mathrm{QD}}\right)\right]$ and $\mathcal{L}_{n, \sigma}^{\mathrm{QD}}=$ $\int_{-\infty}^{\infty} d \omega \omega^{n}(-\partial f / \partial \omega) \mathcal{T}_{\sigma}(\omega)$ for $n=0,1,2$.

[37] T. A. Costi and V. Zlatić, Phys. Rev. B 81, 235127 (2010).

[38] A. C. Hewson, J. Phys. Condens. Matter 13, 10011 (2001).

[39] Y. Nishikawa, D. J. G. Crow, and A. C. Hewson, Phys. Rev. B 82, 245109 (2010).
[40] A. Oguri, Phys. Rev. B 85, 155404 (2012).

[41] K. M. Stadler, A. K. Mitchell, J. von Delft, and A. Weichselbaum, Phys. Rev. B 93, 235101 (2016).

[42] $\bar{C}_{s}^{b}=\left(\pi^{2} / 192\right)\left[\cos \left(2 \pi m_{d}\right)+\left\{4+5 \cos \left(2 \pi m_{d}\right)\right\}(R-1)^{2}+\right.$ $\left.\Theta_{V}^{b} \cos \left(\pi m_{d}\right)\right]\left(T_{K} / T^{*}\right)^{2}, \quad$ and $\quad \Theta_{V}^{b} \equiv-\left[\sin \left(\pi m_{d}\right) / 2 \pi\right]$ $\left[\left(\chi_{\uparrow \uparrow \uparrow}^{[3]}+3 \chi_{\uparrow \downarrow \downarrow}^{[3]}\right) / \chi_{\uparrow \uparrow}^{2}\right]$. 